\title{
Integral Representation for the Electron-Atom Ionization Amplitude which is Free of Ambiguity and Divergence Problems
}

\author{
A. S. Kadyrov, ${ }^{1}$ A. M. Mukhamedzhanov, ${ }^{2}$ A. T. Stelbovics, ${ }^{1}$ and I. Bray ${ }^{1}$ \\ ${ }^{1}$ Centre for Atomic, Molecular and Surface Physics, Division of Science and Engineering, Murdoch University, \\ Perth 6150, Australia \\ ${ }^{2}$ Cyclotron Institute, Texas A\&M University, College Station, Texas 77843, USA
}

(Received 15 May 2003; published 17 December 2003)

\begin{abstract}
It is shown that existing problems with the formal theory of ionization can be effectively resolved. An integral representation for the ionization amplitude free of ambiguity and divergence problems is given. Moreover, the ionization amplitude in the new formulation is shown directly to have an ideal form for practical calculations.
\end{abstract}

DOI: $10.1103 /$ PhysRevLett.91.253202

PACS numbers: $34.10 .+\mathrm{x}$, 03.65.Nk, 34.80.Dp

With the spectacular progress in high-performance computing, the study of electron-atom ionization through various direct numerical calculations has emerged as a powerful approach to the problem (see, e.g., [1]). Agreement with experiment is generally very good. However, otherwise sophisticated approaches to the problem such as exterior complex scaling (ECS) [2], convergent close coupling [3], $R$ matrix [4], $T$ matrix [5], etc., all rely on one or another form of approximation when it comes to extracting the ionization amplitude. Furthermore, the formal theory of ionization is presently not able to show how to calculate the ionization amplitude unambiguously. In this Letter, we give a new integral representation for the ionization amplitude, which is free of ambiguity and divergence problems, and show that this representation is capable of considerably simplifying practical calculations. The approach described here is rather general as is the nature of the problem. The results given below are readily applicable to extract the exact amplitudes in direct calculations of other atomic [6] and nuclear [7] three-body breakup processes. They may also be useful in further developing the effective-charge perturbation approaches [8-10].

Without the loss of generality, we consider here electron-impact ionization of atomic hydrogen and assume that the proton is infinitely heavy compared to the electrons and remains at rest. To avoid inessential complications we also assume the electrons to be distinguishable. The generalization to the case of indistinguishable electrons is straightforward. Atomic units are used throughout.

The ionization amplitude in the prior form is defined according to [11]

$$
T\left(\boldsymbol{k}_{1}, \boldsymbol{k}_{2}\right)=\int d \boldsymbol{r}_{1} d \boldsymbol{r}_{2} \Psi^{-*}\left(\boldsymbol{r}_{1}, \boldsymbol{r}_{2}\right) V_{i} \Phi^{(i)}\left(\boldsymbol{r}_{1}, \boldsymbol{r}_{2}\right) .
$$

Here $\Phi^{(i)}$ is the initial-channel wave function and $\Psi^{-}$is a total wave function describing scattering state of all three particles in the continuum $(e+e+p \rightarrow e+e+p)$ with incoming scattered-wave boundary condition. It satisfies

$$
(E-H) \Psi^{-}\left(\boldsymbol{r}_{1}, \boldsymbol{r}_{2}\right)=0,
$$

where $H=H_{0}+V$ is the three-body Hamiltonian, $H_{0}$ is the free Hamiltonian, $V$ is the full interaction, and $E$ is the total energy of the system. The potential $V_{i}$ in (1) is the interaction of the incident electron with the target particles, $\boldsymbol{r}_{1}$ and $\boldsymbol{r}_{2}$ are the coordinates of the electrons relative to the proton, and $\boldsymbol{k}_{1}$ and $\boldsymbol{k}_{2}$ are their momenta.

The ionization amplitude in the form (1) is not convenient for full-scale practical calculations, for it requires calculation of the total scattering wave function $\Psi^{-}$, which develops from an initial state of three free particles. Furthermore, in this case knowledge of $\Psi^{-}$in the entire space would be necessary.

In searching for more practical ways of calculating the ionization amplitude Peterkop [12,13] and Rudge [14] considered the integral

$$
\begin{aligned}
I_{z_{1}, z_{2}}\left(\boldsymbol{k}_{1}, \boldsymbol{k}_{2}\right)= & \int d \boldsymbol{r}_{1} d \boldsymbol{r}_{2} \Phi^{+}\left(\boldsymbol{r}_{1}, \boldsymbol{r}_{2}\right) \\
& \times(H-E) \Psi^{(2 \mathrm{C})-*}\left(\boldsymbol{r}_{1}, \boldsymbol{r}_{2}\right),
\end{aligned}
$$

where $\Phi^{+}$is a solution of the Schrödinger equation

$$
(E-H) \Phi^{+}\left(\boldsymbol{r}_{1}, \boldsymbol{r}_{2}\right)=0
$$

describing the actual ionization process $(e+H \rightarrow e+$ $e+p$ ) with outgoing scattered-wave boundary condition [15]. The function $\Psi^{(2 \mathrm{C})-}$ is a product of two Coulomb wave functions of effective charges $z_{1}$ and $z_{2}$ :

$$
\Psi^{(2 \mathrm{C})-}\left(\boldsymbol{r}_{1}, \boldsymbol{r}_{2}\right)=e^{i \boldsymbol{k}_{1} \cdot \boldsymbol{r}_{1}} \psi_{z_{1}}\left(\boldsymbol{k}_{1}, \boldsymbol{r}_{1}\right) e^{i \boldsymbol{k}_{2} \cdot \boldsymbol{r}_{2}} \psi_{z_{2}}\left(\boldsymbol{k}_{2}, \boldsymbol{r}_{2}\right),
$$

with incoming wave boundary condition, where

$$
\begin{aligned}
\psi_{\nu}(\boldsymbol{k}, \boldsymbol{r})= & \Gamma(1+i \nu / k) \exp (\pi \nu / 2 k) \\
& \times{ }_{1} F_{1}(-i \nu / k, 1 ;-i(k r+\boldsymbol{k} \cdot \boldsymbol{r})),
\end{aligned}
$$

and where ${ }_{1} F_{1}$ is the confluent hypergeometric function.

Using Eq. (4) and Green's theorem, the volume integral in Eq. (3) can be transformed into a surface integral 


$$
\begin{aligned}
I_{z_{1}, z_{2}}\left(\boldsymbol{k}_{1}, \boldsymbol{k}_{2}\right)= & \frac{1}{2} \lim _{R \rightarrow \infty} R^{5} \int d \hat{\boldsymbol{r}}_{1} d \hat{\boldsymbol{r}}_{2} \int_{0}^{\pi / 2} d \alpha \sin ^{2} \alpha \cos ^{2} \alpha \\
& \times\left[\Psi^{(2 \mathrm{C})-*} \frac{\partial \Phi^{+}}{\partial R}-\Phi^{+} \frac{\partial \Psi^{(2 \mathrm{C})-*}}{\partial R}\right],
\end{aligned}
$$

where $R=\left(r_{1}^{2}+r_{2}^{2}\right)^{1 / 2}$ is the hyperradius and $\left(\hat{\boldsymbol{r}}_{1}, \hat{\boldsymbol{r}}_{2}, \alpha\right)$ is the five-dimensional hyperangle, with $\alpha=\arctan \left(r_{2} / r_{1}\right)$.

Expression (7) is better than (1) in terms of practical calculations, for it is more convenient to calculate the total wave function $\Phi^{+}$being developed from the simple (and exact) initial state $\Phi^{(i)}$ given by a product of a plane wave and a hydrogen bound state wave function. In addition, the expression (7) is amenable to partial-wave expansion, as a result of which it reduces to a sum of one-dimensional integrals. This is in contrast with Eq. (1), which after partial-wave expansion at best reduces to a two-dimensional integral. Finally, we emphasize that $I_{z_{1}, z_{2}}$ depends only on the asymptotic behavior of the wave functions $\Phi^{+}$and $\Psi^{(2 \mathrm{C})-}$ on an infinitely large hypersphere, and therefore, knowledge of the wave functions over the entire space is not required.

The asymptotic behavior of $\Phi^{+}$when all interparticle distances are large (i.e., $r_{1} \rightarrow \infty, r_{2} \rightarrow \infty$, and $r_{3} \rightarrow \infty$, where $\boldsymbol{r}_{3}=\boldsymbol{r}_{1}-\boldsymbol{r}_{2}$; we call this domain $\Omega_{0}$ ) was found by Peterkop [16] and is written as

$$
\Phi^{+}\left(\boldsymbol{r}_{1}, \boldsymbol{r}_{2}\right) \stackrel{\Omega_{0}}{\longrightarrow} A\left(\hat{\boldsymbol{r}}_{1}, \hat{\boldsymbol{r}}_{2}, \alpha\right) R^{-5 / 2} e^{i \kappa R+i \gamma \ln (\kappa R)},
$$

where $\kappa=(2 E)^{1 / 2}$,

$$
\gamma=\frac{1}{\kappa}\left[\frac{1}{\sin \alpha}+\frac{1}{\cos \alpha}-\frac{1}{\sqrt{1-\hat{\boldsymbol{r}}_{1} \cdot \hat{\boldsymbol{r}}_{2} \sin 2 \alpha}}\right],
$$

and $A$ is the ionization amplitude. With this asymptotic form Peterkop demonstrated that $I_{z_{1}, z_{2}}$ exists and differs from the ionization amplitude only by a phase factor

$$
A\left(\hat{\boldsymbol{k}}_{1}, \hat{\boldsymbol{k}}_{2}, \alpha^{\prime}\right)=\frac{\boldsymbol{\kappa}^{3 / 2}}{(2 \pi)^{5 / 2}} e^{i \beta(R)+i \pi / 4} I_{z_{1}, z_{2}}\left(\boldsymbol{k}_{1}, \boldsymbol{k}_{2}\right),
$$

where $\alpha^{\prime}=\arctan \left(k_{2} / k_{1}\right)$. However, Eq. (10) involves a phase factor $\beta$ which diverges as $R \rightarrow \infty$ unless the effective charges satisfy

$$
\frac{z_{1}}{k_{1}}+\frac{z_{2}}{k_{2}}=\frac{1}{k_{1}}+\frac{1}{k_{2}}-\frac{1}{\left|\boldsymbol{k}_{1}-\boldsymbol{k}_{2}\right|},
$$

in which case the phase factor $\beta$ vanishes. Equation (11) is known as the Peterkop condition and Eq. (10) with Eq. (7), or (3), as the integral representation for the ionization amplitude.

Thus, $z_{1}$ and $z_{2}$ depend on vectors $\boldsymbol{k}_{1}$ and $\boldsymbol{k}_{2}$. For this reason this procedure was not very useful in practice for almost 40 years, for it is not clear how to implement condition (11) in realistic calculations. From a practical point of view, problems with Peterkop's effective-charge approach were pointed out recently by Baertschy et al. [2]. Their calculations showed that a hypothetical possibility to find suitable effective charges $z_{1}$ and $z_{2}$ would lead to numerical problems due to nonorthogonality of the Coulomb wave of a nonunit effective charge to the bound states of hydrogen. From the formal side, even if the Peterkop condition is satisfied this would not allow one to establish the ionization amplitude in full. This is because, as mentioned by Peterkop [13], some part of the complex amplitude $A$ in asymptotic form (8) can be moved to the phase factor and the resulting wave function is still a solution to the original Eq. (4) transformed into the six-dimensional hyperspace. Accordingly, the remaining part of $A$ can equally well be called an ionization amplitude.

On the other hand, if Eq. (10) is correct, then due to the fact that cross sections depend only on the magnitude of the amplitude one can ignore the phase part with its ambiguity and divergence problems altogether. This is exactly what is done, for example, in the ECS [2] method. Indeed, the calculations made using Eqs. (10) and (3) with $z_{1}=z_{2}=1$ show generally very good agreement with experiment. So, what is wrong with the formal theory of ionization? Why is one required to ignore obvious problems of the theory in order to proceed with practical calculations, yet the calculations agree well with experiment? In the end, is the procedure based on Eq. (10) and used in the aforementioned approaches correct?

To begin, we consider the Peterkop formulation summarized by Eqs. (10) and (3) and show that it is incomplete. For further discussion we should distinguish all possible geometries where condition $R \rightarrow \infty$ is satisfied. In addition to the $\Omega_{0}$ domain defined earlier, we identify the domain where $r_{1} \rightarrow \infty, r_{2} \rightarrow \infty$ with limited $r_{3}$ as $\Omega_{3}$ and when $r_{2}$ (or $r_{1}$ ) goes to infinity but $r_{1}\left(r_{2}\right)$ remains limited as $\Omega_{2}\left(\Omega_{1}\right)$. Domains $\Omega_{1}, \Omega_{2}$, and $\Omega_{3}$ correspond to $\alpha \rightarrow 0, \alpha \rightarrow \pi / 2$, and $\alpha \rightarrow \pi / 4$ in the above integral, respectively.

The problems with Peterkop's integral representation for the ionization amplitude originate from the fact that the Peterkop asymptotic form used to calculate the integral (7) is invalid in all asymptotic regimes except $\Omega_{0}$. It is clearly seen from Eq. (9) that the Peterkop form cannot be used when $\alpha \rightarrow 0, \alpha \rightarrow \pi / 2$, and $\alpha \rightarrow \pi / 4$. At the same time integration over $\alpha$ runs through all these points. Thus, in the integral representation suggested by Peterkop the contributions from $\Omega_{1}, \Omega_{2}$, and $\Omega_{3}$ domains are either missing or taken into account incorrectly.

To establish the correct relationship between the ionization amplitude and the integral (7), if there is any, we need the full ambiguity-free asymptotic form of the total scattering wave function $\Phi^{+}$. Such an asymptotic form has been given recently $[17,18]$ for each of the mentioned domains separately. A combined result valid in all of these domains is [19] 


$$
\Phi^{+}\left(\boldsymbol{r}_{1}, \boldsymbol{r}_{2}\right) \stackrel{R \rightarrow \infty}{\longrightarrow} \frac{\kappa^{3 / 2}}{(2 \pi)^{5 / 2}} T\left(\frac{\kappa}{R} \boldsymbol{r}_{1}, \frac{\kappa}{R} \boldsymbol{r}_{2}\right) \frac{1}{R^{5 / 2}} e^{i \kappa R+i \pi / 4} \psi_{1}\left(\boldsymbol{K}_{1}, \boldsymbol{r}_{1}\right) \psi_{1}\left(\boldsymbol{K}_{2}, \boldsymbol{r}_{2}\right) \psi_{-1 / 2}\left(\boldsymbol{K}_{3}, \boldsymbol{r}_{3}\right)
$$

where

$$
\boldsymbol{K}_{1}=\frac{\kappa}{R} \boldsymbol{r}_{1}+\frac{R}{\kappa r_{3}^{3}} \boldsymbol{r}_{3}, \quad \boldsymbol{K}_{2}=\frac{\kappa}{R} \boldsymbol{r}_{2}+\frac{R}{\kappa r_{3}^{3}} \boldsymbol{r}_{3}, \quad \boldsymbol{K}_{3}=\frac{\kappa}{2 R} \boldsymbol{r}_{3}-\frac{R}{\kappa}\left(\frac{\boldsymbol{r}_{1}}{r_{1}^{3}}+\frac{\boldsymbol{r}_{2}}{r_{2}^{3}}\right) .
$$

In (12) the ionization amplitude $T$ is as defined according to Eq. (1) (see [17,18]) and therefore unambiguous.

Then for the surface integral in (7) we get

$$
\begin{aligned}
I_{z_{1}, z_{2}}\left(\boldsymbol{k}_{1}, \boldsymbol{k}_{2}\right)= & \frac{i}{2} \lim _{R \rightarrow \infty} R^{5} \int d \hat{\boldsymbol{r}}_{1} d \hat{\boldsymbol{r}}_{2} \int_{0}^{\pi / 2} d \alpha \sin ^{2} \alpha \cos ^{2} \alpha\left[\kappa+\cos \alpha \boldsymbol{k}_{1} \cdot \hat{\boldsymbol{r}}_{1}+\sin \alpha \boldsymbol{k}_{2} \cdot \hat{\boldsymbol{r}}_{2}\right] \Phi^{+}\left(R \cos \alpha \hat{\boldsymbol{r}}_{1}, R \sin \alpha \hat{\boldsymbol{r}}_{2}\right) \\
& \times \exp \left[-i R \cos \alpha \boldsymbol{k}_{1} \cdot \hat{\boldsymbol{r}}_{1}-i R \sin \alpha \boldsymbol{k}_{2} \cdot \hat{\boldsymbol{r}}_{2}\right] \psi_{z_{1}}^{*}\left(\boldsymbol{k}_{1}, R \cos \alpha \hat{\boldsymbol{r}}_{1}\right) \psi_{z_{2}}^{*}\left(\boldsymbol{k}_{2}, R \sin \alpha \hat{\boldsymbol{r}}_{2}\right) .
\end{aligned}
$$

Using the asymptotic form of the plane wave we immediately have

$$
\begin{aligned}
I_{z_{1}, z_{2}}\left(\boldsymbol{k}_{1}, \boldsymbol{k}_{2}\right)= & \frac{2 \pi^{2}}{i k_{1} k_{2}} \lim _{R \rightarrow \infty} R^{3} \int_{0}^{\pi / 2} d \alpha \sin \alpha \cos \alpha \\
& \times\left\{\left[\kappa+k_{1} \cos \alpha+k_{2} \sin \alpha\right] \Phi^{+}\left(R \cos \alpha \hat{\boldsymbol{k}}_{1}, R \sin \alpha \hat{\boldsymbol{k}}_{2}\right) \exp \left[-i R k_{1} \cos \alpha-i R k_{2} \sin \alpha\right]\right. \\
& \quad \times \psi_{z_{1}}^{*}\left(\boldsymbol{k}_{1}, R \cos \alpha \hat{\boldsymbol{k}}_{1}\right) \psi_{z_{2}}^{*}\left(\boldsymbol{k}_{2}, R \sin \alpha \hat{\boldsymbol{k}}_{2}\right)-\left[\kappa-k_{1} \cos \alpha-k_{2} \sin \alpha\right] \Phi^{+}\left(-R \cos \alpha \hat{\boldsymbol{k}}_{1},-R \sin \alpha \hat{\boldsymbol{k}}_{2}\right) \\
& \left.\quad \times \exp \left[i R k_{1} \cos \alpha+i R k_{2} \sin \alpha\right] \psi_{z_{1}}^{*}\left(\boldsymbol{k}_{1},-R \cos \alpha \hat{\boldsymbol{k}}_{1}\right) \psi_{z_{2}}^{*}\left(\boldsymbol{k}_{2},-R \sin \alpha \hat{\boldsymbol{k}}_{2}\right)+\cdots\right\} .
\end{aligned}
$$

This is an extremely oscillatory integral as $R \rightarrow \infty$. Therefore, only points of stationary phase in $\alpha$ will contribute to the integral. One can verify that both terms within the braces have a common stationary-phase point at $k_{1} \sin \alpha=k_{2} \cos \alpha$, where we have $\cos \alpha=k_{1} / \kappa$ and $\sin \alpha=k_{2} / \kappa$. We see then that the second term is identically zero at the stationary point. The dots indicate other terms of the integrand, which have no stationary points and, therefore, do not contribute to the integral. Calculating the remaining integral, we arrive at

$$
I_{z_{1}, z_{2}}\left(\boldsymbol{k}_{1}, \boldsymbol{k}_{2}\right)=T\left(\boldsymbol{k}_{1}, \boldsymbol{k}_{2}\right) \lim _{R \rightarrow \infty} \tau_{z_{1}, z_{2}}\left(\boldsymbol{k}_{1}, \boldsymbol{k}_{2}, R\right)
$$

where

$$
\begin{aligned}
\tau_{z_{1}, z_{2}}\left(\boldsymbol{k}_{1}, \boldsymbol{k}_{2}, R\right)= & \psi_{z_{1}}^{*}\left(\boldsymbol{k}_{1}, R \boldsymbol{k}_{1} / \kappa\right) \psi_{z_{2}}^{*}\left(\boldsymbol{k}_{2}, R \boldsymbol{k}_{2} / \kappa\right) \\
& \times \psi_{1}\left(\tilde{\boldsymbol{K}}_{1}, R \boldsymbol{k}_{1} / \kappa\right) \psi_{1}\left(\tilde{\boldsymbol{K}}_{2}, R \boldsymbol{k}_{2} / \kappa\right) \\
& \times \psi_{-1 / 2}\left(\tilde{\boldsymbol{K}}_{3}, 2 R \boldsymbol{k}_{3} / \boldsymbol{\kappa}\right),
\end{aligned}
$$

and

$$
\begin{gathered}
\tilde{\boldsymbol{K}}_{1}=\boldsymbol{k}_{1}+\frac{\kappa}{4 R} \frac{\boldsymbol{k}_{3}}{k_{3}^{3}}, \quad \tilde{\boldsymbol{K}}_{2}=\boldsymbol{k}_{2}+\frac{\kappa}{4 R} \frac{\boldsymbol{k}_{3}}{k_{3}^{3}}, \\
\tilde{\boldsymbol{K}}_{3}=\boldsymbol{k}_{3}-\frac{\kappa}{R}\left(\frac{\boldsymbol{k}_{1}}{k_{1}^{3}}+\frac{\boldsymbol{k}_{2}}{k_{2}^{3}}\right),
\end{gathered}
$$

with $\boldsymbol{k}_{3}=\left(\boldsymbol{k}_{1}-\boldsymbol{k}_{2}\right) / 2$.

First of all, we see that $I_{z_{1}, z_{2}}$ is $R$ dependent no matter what $z_{1}$ and $z_{2}$ are or how large $R$ is. So it is appropriate to write $I_{z_{1}, z_{2}}\left(\boldsymbol{k}_{1}, \boldsymbol{k}_{2}\right) \equiv I_{z_{1}, z_{2}}\left(\boldsymbol{k}_{1}, \boldsymbol{k}_{2}, R\right)$. Second, from (16) it turns out that one can indeed represent the ionization amplitude in terms of the trial integral (7); however, the correct relationship is written as

$$
T\left(\boldsymbol{k}_{1}, \boldsymbol{k}_{2}\right)=\lim _{R \rightarrow \infty} \frac{I_{z_{1}, z_{2}}\left(\boldsymbol{k}_{1}, \boldsymbol{k}_{2}, R\right)}{\tau_{z_{1}, z_{2}}\left(\boldsymbol{k}_{1}, \boldsymbol{k}_{2}, R\right)} .
$$

Furthermore, $\tau_{z_{1}, z_{2}}$ is well behaved in the entire space for arbitrary $z_{1}$ and $z_{2}$. Consequently, there is no necessity for $z_{1}$ and $z_{2}$ to satisfy a particular condition in contrast to condition (11) required for the Peterkop amplitude (10). Therefore, one may choose $z_{1}$ and $z_{2}$ such that they maximally simplify the practical calculation of $I_{z_{1}, z_{2}}$. The resulting ionization amplitude (19) will not depend on this choice.

Thus, the ionization amplitude can be represented in terms of a trial integral which has a structure ideally suited for practical calculations. However, we can go even further and ask the question: Is it not possible, if our understanding of the formal theory is complete, to extract the ionization amplitude without recourse to external trial quantities? The answer is yes, as we now demonstrate. Consider the following expression:

$$
I\left(\boldsymbol{k}_{1}, \boldsymbol{k}_{2}\right)=\left\langle\Psi^{-}\left|\overleftarrow{\mathrm{H}}_{0}-\overrightarrow{\mathrm{H}}_{0}\right| \Phi^{(\mathrm{sc})+}\right\rangle
$$

where $\Phi^{(\mathrm{sc})+}=\Phi^{+}-\Phi^{(i)}$ is the scattered part of the total wave function $\Phi^{+}$. A left (right) arrow on the differential Hamiltonian operator indicates that it acts on the bra (ket) state. Equation (20) is also convenient for numerical calculations as it can easily be transformed to a surface integral similar to (7). In order to calculate this integral, in addition to the asymptotic form of $\Phi^{(\mathrm{sc})+}$ given by Eq. (12), we also need an equivalent form of 
the wave function $\Psi^{-}$which has been given in Ref. [20]. Transforming Eq. (20) into a surface integral and calculating it making use of the correct asymptotic behavior for $\Psi^{-}$and $\Phi^{(\mathrm{sc})+}$, we get after some algebra

$$
\begin{aligned}
I\left(\boldsymbol{k}_{1}, \boldsymbol{k}_{2}\right)= & T\left(\boldsymbol{k}_{1}, \boldsymbol{k}_{2}\right) \lim _{R \rightarrow \infty}\left|\psi_{1}\left(\tilde{\boldsymbol{K}}_{1}, R \boldsymbol{k}_{1} / \boldsymbol{\kappa}\right)\right|^{2} \\
& \times\left|\psi_{1}\left(\tilde{\boldsymbol{K}}_{2}, R \boldsymbol{k}_{2} / \kappa\right)\right|^{2}\left|\psi_{-1 / 2}\left(\tilde{\boldsymbol{K}}_{3}, 2 R \boldsymbol{k}_{3} / \kappa\right)\right|^{2} .
\end{aligned}
$$

By virtue of the three-body correlation effects, $\tilde{\boldsymbol{K}}_{1}, \tilde{\boldsymbol{K}}_{2}$, and $\tilde{\boldsymbol{K}}_{3}$ do not vanish even in kinematical regimes where their respective driving terms $\boldsymbol{k}_{1}, \boldsymbol{k}_{2}$, and $\boldsymbol{k}_{3}$ are close to zero. Therefore, we can safely take the remaining limit to obtain

$$
I\left(\boldsymbol{k}_{1}, \boldsymbol{k}_{2}\right)=T\left(\boldsymbol{k}_{1}, \boldsymbol{k}_{2}\right) .
$$

Thus, Eq. (20) is nothing but a surface-integral form of the exact ionization amplitude itself.

This remarkable result can be verified independently as follows. First note that Eq. (4) can be written as

$$
(E-H) \Phi^{(\mathrm{sc})+}\left(\boldsymbol{r}_{1}, \boldsymbol{r}_{2}\right)=V_{i} \Phi^{(i)}\left(\boldsymbol{r}_{1}, \boldsymbol{r}_{2}\right) .
$$

Combining this with Eq. (2), it immediately follows that

$$
\begin{aligned}
T\left(\boldsymbol{k}_{1}, \boldsymbol{k}_{2}\right) & \equiv\left\langle\Psi^{-}\left|V_{i}\right| \Phi^{(i)}\right\rangle=\left\langle\Psi^{-}|E-\overrightarrow{\mathrm{H}}| \Phi^{(\mathrm{sc})+}\right\rangle \\
& =\left\langle\Psi^{-}|\overleftarrow{\mathrm{H}}-E| \Phi^{(\mathrm{sc})+}\right\rangle+\left\langle\Psi^{-}|E-\overrightarrow{\mathrm{H}}| \Phi^{(\mathrm{sc})+}\right\rangle \\
& =\left\langle\Psi^{-}\left|\overleftarrow{\mathrm{H}}_{0}-\overrightarrow{\mathrm{H}}_{0}\right| \Phi^{(\mathrm{sc})+}\right\rangle \equiv I\left(\boldsymbol{k}_{1}, \boldsymbol{k}_{2}\right) .
\end{aligned}
$$

This is truly a powerful demonstration of the selfconsistency of the theory when it is formulated in a mathematically correct fashion. The latter also makes it obvious that the amplitude of any fragmentation process in a many-body system can be directly written in a convenient surface-integral form.

What are the consequences of the present formulation when practical calculations are considered? The analytically given $\tau_{z_{1}, z_{2}}$ is increasingly oscillatory with increasing $R$. Therefore, $I_{z_{1}, z_{2}}$ presently being used in large-scale computations must be increasingly oscillatory as well. The use of Eq. (19) instantly washes away these unnecessary oscillations from $I_{z_{1}, z_{2}}$ leaving only the exact amplitude. This should simplify the calculations immensely. The cross sections calculated with Eq. (19) are expected to vary somewhat in the kinematical regimes where any of $k_{1}, k_{2}$, or $k_{3}$ are small compared to the cross sections calculated from $I_{z_{1}, z_{2}}$ alone. In all other kinematics, where $\left|\tau_{z_{1}, z_{2}}\right| \rightarrow 1$ with increasing $R$, the present formulation should correct the phase problems with the amplitude without significantly changing its magnitude. Our analysis thus provides a formal justification of the cross sections obtained in the ECS method [1,2], in such kinematical regimes.

Summarizing, we have been able to resolve longstanding problems in the theory of ionization and pro- vided a new indirect, but practical, prescription for calculating ionization amplitudes. We have also shown that in the new formulation the ionization amplitude directly takes a surface-integral form ideal for practical calculations. The presented theory is readily applicable to extract the exact breakup amplitudes in direct calculations of general few-body systems spanning atomic, molecular, and nuclear physics.

This work was supported by the Australian Research Council, U.S. DOE Grant No. DE-FG03-93ER40773 and NSF Grant No. PHY-0140343.

[1] T. N. Rescigno, M. Baertschy, W. A. Isaacs, and C.W. McCurdy, Science 286, 2474 (1999).

[2] M. Baertschy, T. N. Rescigno, and C.W. McCurdy, Phys. Rev. A 64, 022709 (2001).

[3] I. Bray, D.V. Fursa, A. S. Kheifets, and A. T. Stelbovics, J. Phys. B 35, R117 (2002).

[4] K. Bartschat, E. T. Hudson, M. P. Scott, P. G. Burke, and V. M. Burke, J. Phys. B 29, 115 (1996).

[5] M. S. Pindzola, D. Mitnik, and F. Robicheaux, Phys. Rev. A 62, 062718 (2000).

[6] L. Malegat, P. Selles, and A. Kazansky, Phys. Rev. Lett. 85, 4450 (2000).

[7] A. Kievsky, S. Rosati, and M. Viviani, Phys. Rev. Lett. 82, 3759 (1999).

[8] C. Pan and A. F. Starace, Phys. Rev. Lett. 67, 185 (1991).

[9] M. Pont, R. Shakeshaft, F. Maulbetsch, and J. S. Briggs, Phys. Rev. A 53, 3671 (1996).

[10] J. Berakdar and J. S. Briggs, Phys. Rev. Lett. 72, 3799 (1994).

[11] R. G. Newton, in Scattering Theory of Waves and Particles, Texts and Monographs in Physics (Springer-Verlag, New York, 1982), 2nd ed.

[12] R. K. Peterkop, Opt. Spektrosk. 13, 153 (1962) [Opt. Sectrosc. 13, 87 (1962)].

[13] R. K. Peterkop, Theory of Ionization of Atoms by Electron-Impact (Colorado Associated University Press, Boulder, 1977).

[14] M. R. H. Rudge, Rev. Mod. Phys. 40, 564 (1968).

[15] Clearly, wave functions $\Psi^{-}$and $\Phi^{+}$are forms of the same total wave function of the three-body system, satisfying two different boundary conditions.

[16] R. K. Peterkop, Izv. Akad. Nauk Latv. SSR, Riga 9, 79 (1960).

[17] A. S. Kadyrov, A. M. Mukhamedzhanov, A. T. Stelbovics, I. Bray, and F. Pirlepesov, Phys. Rev. A 68, 022703 (2003).

[18] A. S. Kadyrov, A. M. Mukhamedzhanov, and A.T. Stelbovics, Phys. Rev. A 67, 024702 (2003).

[19] We drop here two-cluster parts of the asymptotic wave function, present in $\Omega_{1}$ and $\Omega_{2}$ domains; we will, however, show elsewhere that they do not contribute to the integral we are considering.

[20] E. O. Alt and A. M. Mukhamedzhanov, Phys. Rev. A 47, 2004 (1993). 